\title{
Dynamics of ordering in highly degenerate models with anisotropic grain-boundary potential: Effects of temperature and vortex formation
}

Jeppesen, Claus; Flyvbjerg, Henrik; Mouritsen, Ole G.

Published in:

Physical Review B

Link to article, DOI:

10.1103/PhysRevB.40.9070

Publication date:

1989

Document Version

Publisher's PDF, also known as Version of record

Link back to DTU Orbit

Citation (APA):

Jeppesen, C., Flyvbjerg, H., \& Mouritsen, O. G. (1989). Dynamics of ordering in highly degenerate models with anisotropic grain-boundary potential: Effects of temperature and vortex formation. Physical Review $B, 40(13)$, 9070-9079. https://doi.org/10.1103/PhysRevB.40.9070

\section{General rights}

Copyright and moral rights for the publications made accessible in the public portal are retained by the authors and/or other copyright owners and it is a condition of accessing publications that users recognise and abide by the legal requirements associated with these rights.

- Users may download and print one copy of any publication from the public portal for the purpose of private study or research.

- You may not further distribute the material or use it for any profit-making activity or commercial gain

- You may freely distribute the URL identifying the publication in the public portal 


\title{
Dynamics of ordering in highly degenerate models with anisotropic grain-boundary potential: Effects of temperature and vortex formation
}

\author{
Claus Jeppesen and Henrik Flyvbjerg \\ Niels Bohr Institute, University of Copenhagen, Blegdamsvej 17, DK-2100 Copenhagen $\emptyset$ Denmark \\ Ole G. Mouritsen \\ Department of Structural Properties of Materials, The Technical University of Denmark, Building 307 \\ DK-2800 Lyngby, Denmark
}

(Received 16 March 1989)

\begin{abstract}
Monte Carlo computer-simulation techniques are used to elucidate the equilibrium phase behavior as well as the late-stage ordering dynamics of some two-dimensional models with ground-state ordering of a high degeneracy, $Q$. The models are $Q$-state Potts models with anisotropic grain-boundary potential on triangular lattices - essentially clock models, except that the potential is not a cosine, but a sine function of the angle between neighboring grain orientations. For not too small $Q$, these models display two thermally driven phase transitions, one which takes the system from a low-temperature Potts-ordered phase to an intermediate phase which lacks conventional long-range order, and another transition which takes the system to the high-temperature disordered phase. The linear nature of the sine potential used makes it a marginal case in the sense that it favors neither hard domain boundaries, like the standard Potts models do, nor a wetting of the boundaries, as the standard clock models do. Thermal fluctuations nevertheless cause wetting to occur for not too small temperatures. Specifically, we have studied models with $Q=12$ and 48 . The models are quenched from infinite to zero as well as finite temperatures within the two low-temperature phases. The order parameter is a nonconserved quantity during these quenches. The nonequilibrium ordering process subsequent to the quench is studied as a function of time by calculating the interfacial energy, $\Delta E$, associated with the entire grain-boundary network. The time evolution of this quantity is shown to obey the growth law, $\Delta E(t) \sim t^{-n}$, over an extended time range at late times. It is found that the zerotemperature dynamics is characterized by a special exponent value which for the $Q=48$ model is $n \simeq 0.25$ in accordance with earlier work. However, for quenches to finite temperatures in the Potts-ordered phase there is a distinct crossover to the classical Lifshitz-Allen-Cahn exponent value, $n=\frac{1}{2}$, for both values of $Q$. This supports the conjecture that the zero-temperature dynamics for models with soft domain boundaries belong to a special universality class, and that all models with nonconserved order parameter, independent of ordering degeneracy and softness and origin of domain boundaries, obey the classical growth law at finite temperatures. In quenches to the Potts-ordered phase vortices and antivortices occur and annihilate mutually without pinning the ordering process. The ordering dynamics for quenches into the intermediate phase is also found to be described by an effectively algebraic growth law.
\end{abstract}

\section{INTRODUCTION}

There is now significant theoretical evidence that the dynamical processes of ordering in condensed matter obey dynamical scaling ${ }^{1}$ and mainly fall into two dynamical universality classes. ${ }^{1-8}$ These classes, which are distinguished by the order parameter being a conserved quantity or not, are characterized by different values of the kinetic growth exponent, $n$, of the growth law

$$
R(t) \sim t^{n}
$$

where $R(t)$ is a linear measure of the growing domains. The first universality class is that of model $A$ (in the classification scheme of Halperin and Hohenberg ${ }^{9}$ ) in which the order parameter is not conserved and where it has been found that $n=\frac{1}{2}$ independent of the degeneracy, $p$, of the ordering, $, 5,10-20$ independent of additional conservation laws, ${ }^{18}$ independent of details of the interaction potential, ${ }^{14}$ and even independent of the spatial dimension. ${ }^{19,21}$ The second class [that of model $B$ (Ref. 9)], in which the order parameter is a conserved quantity, has been investigated much less thoroughly. However, recent computer-simulation work ${ }^{3,4,22-26}$ has produced strong evidence in favor of an exponent value $n=\frac{1}{3}$, again independent of details of the dynamical model ${ }^{24,25}$ (e.g., whether it is the Langevin model, the Cahn-Hilliard model, or the kinetic Ising model) and independent of the spatial dimension. ${ }^{22,23,25}$ For this class, less is known about the possible dependence on the ordering degeneracy. ${ }^{27}$ Very recently ${ }^{6}$ some 
new information has also been gained about dynamical scaling and ordering dynamics in tricritical systems with coupled order parameters, one of which is conserved and the other of which is not (model $C$ ). This information is consistent with $n=\frac{1}{3}$. Careful experimental work on two-dimensional ${ }^{28,29}$ and three-dimensional ${ }^{30-32}$ realizations of these universality classes are generally interpreted to be consistent with the theoretical findings. It is somewhat surprising to note that the exponent values $n=\frac{1}{2}$ and $\frac{1}{3}$ are precisely those predicted by the early classical theories of Lifshitz, ${ }^{33}$ Allen and Cahn, ${ }^{34}$ and Lifshitz and Slyozov ${ }^{35}$ using very basic assumptions about the driving forces for the ordering processes (for a review, see Ref. 1).

In reality, the picture of a universal classification of ordering dynamics is, unfortunately, much less clear than that just outlined. This is true of many model theoretical studies as well as a variety of experimental studies. The reasons for this are manifold. On the theoretical model side the picture is often obscured by crossover effects in either temperature ${ }^{15,36,37}$ or in some model parameter. ${ }^{14,18,38}$ Specific examples include crossover to low-temperature freezing-in ${ }^{14,18,36-38}$ or zero-temperature soft-wall dynamics. ${ }^{15,39,40}$ Experimentally, the growth exponent values may be influenced by similar effects in addition to effects due to imperfections and impurities. ${ }^{41}$ At the present stage of a theoretical development, where a definitive theory still does not exist, and where the major theoretical evidence, at least for highly degenerate systems, comes from computersimulation studies, it is therefore important to take the simulation studies beyond the level of the most simple models. Firstly, this is important in order to further test the current universal picture and maybe more importantly to guide the development of a theory. Secondly, studies of more complicated models constitute an important step towards an understanding of more materialspecific problems.

We have performed a Monte Carlo computersimulation study of the ordering dynamics (or grain growth) in two dimensions in a particular highly degenerate $Q$-state Potts model with anisotropic grain-boundary potential. This model was first proposed by Grest et $a l^{42,43}$ as a model of grain growth in polycrystalline aggregates. From Monte Carlo simulations these authors found that for quenches to zero temperature the model potential for large $Q$ leads to a growth law, Eq. (1), with $n \simeq 0.25$, and argued that this is consistent with similar findings for other models ${ }^{39}$ that are capable of supporting "soft" grain boundaries, i.e., domain walls of a finite thickness. The anisotropic Potts model for large $Q$ leads at zero temperature to such broad interfaces made up of a conglomerate of very small Potts-ordered domains. ${ }^{42,43}$ Several authors ${ }^{12,15,40}$ have, however, recently reported computer-simulation results on the finite-temperature dynamics of a variety of soft-wall models which show that the exponent value $n \simeq 0.25$ is at most only valid at zero temperature and that the classical Lifshitz-Allen-Cahn law with $n=\frac{1}{2}$ is recovered, independent of the value of $Q$, as soon as the thermal fluctuations are allowed for. It is therefore obviously of interest to extend the study of the dynamics of the high- $Q$ Potts model with anisotropic grain-boundary potential to nonzero temperature quenches.

The results of the present work show that also the anisotropic grain-boundary potential leads to ordering dynamics with $n \simeq \frac{1}{2}$ at finite temperatures. This finding has important consequences for the interpretation of experimental data for grain growth in polycrystalline aggregates in terms of an anisotropic high- $Q$ Potts model. Furthermore, our model study demonstrates that the morphology of the growing domains is dramatically different at zero and nonzero temperatures. At zero temperature the domain pattern is characterized by compact Potts-ordered domains bound by a network of fairly straight domain boundaries merging predominantly in three-line vertices. However, at low but finite temperatures, the morphology is completely different with formation of distinct vortices and antivortices. From equilibrium Monte Carlo calculations we show that for large $Q$, specifically $Q=12$ and 48 , the model potential leads to two phase transitions, which separate an intermediate phase from a low-temperature Potts-ordered phase and a high-temperature disordered phase. These results are consistent with similar results for the standard $Q$ state clock models ${ }^{44-47}$ for which it has been shown ${ }^{44}$ that an intermediate Kosterlitz-Thouless phase arises for $Q \geq 5$. Finite-temperature quenches into the lowtemperature phase show, again consistent with work on isotropic high- $Q$ Potts models ${ }^{45-47}$ and vortex-forming axial next-nearest-neighbor Ising models ${ }^{16}$ that the kinetic growth exponent is close to $\frac{1}{2}$ in both phases and independent of temperature.

The outline of the paper is as follows: in Sec. II we present the microscopic interaction model and the model dynamics. Section III is devoted to a description of the equilibrium phase behavior of the $Q=12$ and 48 models. The results for the nonequilibrium ordering dynamics are presented in Sec. IV and analyzed in terms of an algebraic growth law. The paper is concluded by a discussion in Sec. V.

\section{MODEL}

\section{A. Hamiltonian}

The model is a $Q$-state Potts model arrayed on a triangular lattice with a pair potential

$$
V_{i j}=J\left|\sin \left(\frac{\pi\left(S_{i}-S_{j}\right)}{Q}\right)\right|,
$$

defined in terms of the Potts variables $S_{i}, S_{i}$ $=1,2, \ldots, Q . J$ is a positive interaction constant. With an interaction Hamiltonian

$$
\mathcal{H}=-\sum_{i>j}\left(J-V_{i j}\right)
$$

this model has been proposed ${ }^{42,43}$ to provide a descrip- 
tion of the grain-boundary energy in polycrystalline aggregates. In that context, $S_{i}$ labels the possible orientations of a microcrystalline domain or grain. The interactions of Eq. (3) are restricted to nearest neighbors on the triangular lattice. The model implies a $Q$-fold degenerate ground-state ordering (Potts ordering) at low temperatures.

The Hamiltonian in Eq. (3) defines two energy scales: its range $J$, and its gap $J \sin (\pi / Q)$. In this respect it is similar to and may be considered a variant of the standard $Q$-state clock models. Hence it is expected ${ }^{44}$ that the equilibrium phase behavior of the model for large $Q$ ( $Q \geq 5$ for the standard clock model) involves two thermal phase transitions: one at $T_{1}$ from a low-temperature Potts-ordered phase to an intermediate phase, and another one at $T_{2}$ from this intermediate phase to the disordered phase. $T_{1}$ is expected to depend on $Q$ as the gap in the link energy; $T_{2}$ is expected to depend only on the range $J$ of the link energy and hence to be independent of $Q$. Thus for the standard clock model

$$
k_{B} T_{1} / J \sim 1-\cos (2 \pi / Q) \sim Q^{-2},
$$

and for its variant studied here, Eq. (2), yields

$$
k_{B} T_{1} / J \sim \sin (\pi / Q) \sim Q^{-1}
$$

For both clock models $k_{B} T_{2} / J \sim 1$.

\section{B. Model dynamics}

The dynamical model used is a local single-site Glauber excitation mechanism that does not conserve the order parameter. The dynamical model is implemented using Monte Carlo techniques. ${ }^{48,49}$ At zero temperature, the specific Monte Carlo method is built on the conventional Metropolis criterion, which accepts trial moves if $\Delta E \leq 0$. A trial move involves the assignment of a randomly chosen Potts state to the site under consideration. If unsuccessful, up to six new trial moves are attempted at the same site before proceeding to the next site. For finite temperatures, we have used the heat-bath method: ${ }^{50}$ the variable on site $i$ is updated to the value $S_{i}$ with probability

$$
P_{i}\left(S_{i}\right)=\frac{\exp \left(\sum_{j}-\left(J-V_{i j}\right) / k_{B} T\right)}{z_{i}},
$$

where $z_{i}$ is a normalization constant, i.e.,

$$
1=\sum_{S_{i}=1}^{Q} P_{i}\left(S_{i}\right)
$$

In Eq. (4) the summation is over sites $j$, which are nearest neighbors to site $i$.

The dynamical model is implemented on a vector processor using a "checkerboard" updating algorithm: the triangular lattice is divided into three identical sublattices such that any site on any sublattice is surrounded by sites only from the other two sublattices. Toroidal boundary conditions are imposed. The three sublattices are updated one after another, and the updating of sites in one sublattice may be viewed as simultaneous. The time is measured in units of Monte Carlo steps per site (MCS/S).

We have studied the model in Eqs. (2) and (3) for $Q=12$ and for $Q=48$. The dynamics of the ordering processes in both models is simulated at zero and at nonzero temperature. The ordering processes have previously been studied in the $(Q=48)$-state model by Grest et al., ${ }^{42,43}$ but only at zero temperature. As a prerequisite to the studies of finite-temperature dynamics we have studied the equilibrium phase behavior of both models.

\section{EQUILIBRIUM PHASE BEHAVIOR}

Figure 1 shows Monte Carlo data for the internal energy per site, $E(T)=\langle\mathcal{H}\rangle / N$, and the specific heat per site, $C(T)$, for the $(Q=12)$-state model on lattices with $N=L \times L$ sites. Data for three different system sizes, $N=15^{2}, 30^{2}$, and $60^{2}$ are shown in order to illustrate finite-size effects. The specific heat is calculated from the fluctuation theorem,

$$
C(T)=\left(N k_{B} T^{2}\right)^{-1}\left(\left\langle\mathcal{H}^{2}\right\rangle-\langle\mathcal{H}\rangle^{2}\right) .
$$

This function clearly exhibits two peaks signaling two phase transitions. The size dependence of these peaks is

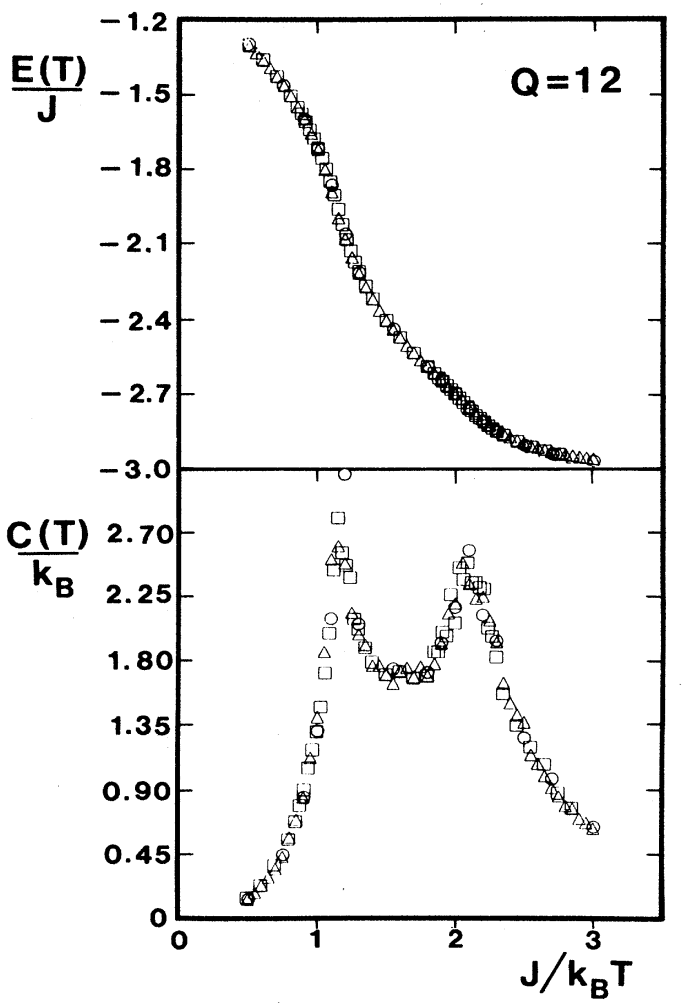

FIG. 1. Equilibrium internal energy per site, $E(T)$, and specific heat per site, $C(T)$, for the $(Q=12)$-state anisotropic Potts model. The data are obtained from Monte Carlo simulations on triangular lattices with $N$ sites. $\square: N=15^{2}, \triangle$ : $N=30^{2}$, and o: $N=60^{2}$. 
weak. For the high-temperature transition there is, however, a systematic increase in peak intensity and shift towards lower temperatures as the system size is increased. The corresponding energy and specific-heat data for the $(Q=48)$-state model are shown in Fig. 2. This model also has two distinct peaks in the specific heat.

The specific-heat data presented in Figs. 1 and 2 are strong numerical indications of two thermal phase transitions in both the $(Q=12)$-state and the $(Q=48)$-state models. The low-temperature (cold) phase in each model is a Potts-ordered phase in which the system is uniformly long-range ordered in one of the $Q$ Potts states. We have found that the intermediate phase of the models we have studied is one with a finite density of tightly bound vortices and antivortices, as it is for the standard clock model. ${ }^{44}$

Although the specific-heat peaks in Figs. 1 and 2 are strong signals of phase transitions, there is no simple relation between the peak positions and the corresponding transition temperatures. ${ }^{51}$ It is known in general to be a very difficult problem to accurately determine the transition temperatures related to Kosterlitz-Thouless phases from computer simulation data. ${ }^{4,51-53}$ For dynamical studies, however, we need only know the approximate location of the phase boundaries, so we are satisfied with the results shown in Figs. 1 and 2, more so because the weak size dependence of $C(T)$ found for these mod-

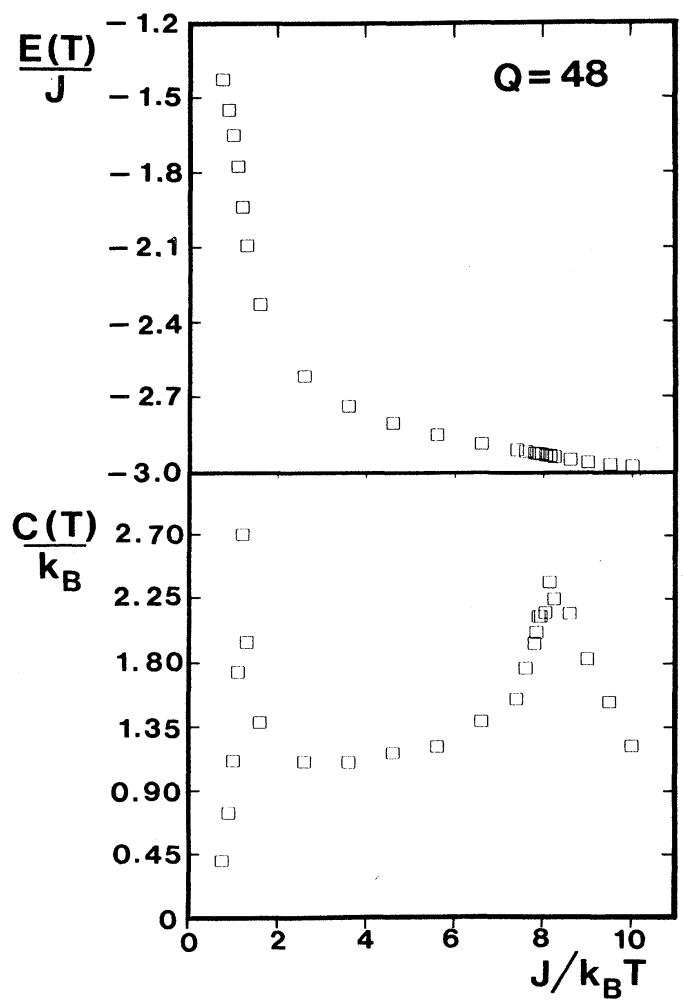

FIG. 2. Equilibrium internal energy per site, $E(T)$, and specific heat per site, $C(T)$, for the $(Q=48)$-state anisotropic Potts model. The data are obtained from Monte Carlo simulations on a triangular lattice with $N=30^{2}$ sites. els is similar to that encountered in the standard clock models, ${ }^{51,52}$ and a comprehensive Monte Carlo study of the $(Q=6)$-state clock model ${ }^{51}$ has shown that the size dependence moves the two specific-heat peaks towards each other, as the system size is increased. We shall assume that this result also applies to the Potts models studied here, so that the stable temperature region for the intermediate phase is less than that suggested by the peaks in Figs. 1 and 2.

If, for a moment, we neglect these subtleties, we may identify the loci of the specific-heat peaks in Figs. 1 and 2 with $T_{1}$ and $T_{2}$. That gives us

$$
J / k_{B} T_{1}(Q=12)=2.1
$$

and

$$
J / k_{B} T_{1}(Q=48)=8.1,
$$

so that

$$
T_{1}(Q=12) / T_{1}(Q=48)=3.9,
$$

to be compared with

$$
[\sin (\pi / 12)] /[\sin (\pi / 48)]=4.0 .
$$

Taking the errors in $T_{1}$ into account, these two ratios are identical, and we see that $T_{1}$ depends on $Q$ as expected. Also as expected, we find that $T_{2}$ is independent of $Q$, since

$$
\begin{gathered}
J / k_{B} T_{2}(Q=12) \simeq J / k_{B} T_{2}(Q=48)=1.17 . \\
\text { IV. NONEQUILIBRIUM ORDERING } \\
\text { DYNAMICS }
\end{gathered}
$$

\section{NONEQUILIBRIUM ORDERING}

The dynamics of the ordering processes is studied subsequent to temperature quenches from infinite temperature to some temperature, $T$, within the ordered phases. The initial state is a disordered, completely random configuration. The time evolution of the system is followed qualitatively by monitoring time sequences of snapshots of microconfigurations. To illustrate the evolution of the morphology of the domain pattern, only the network of interfaces between the domains is studied. Such a study is facilitated by mapping the domain boundaries onto the dual lattice, i.e., onto a honeycomb structure for the triangular lattice. The growth process may be analyzed more quantitatively by calculating the excess nonequilibrium energy

$$
\Delta E(t)=\mathcal{H}(t) / N-E(T)
$$

associated with the entire network of interfaces between the differently ordered domains. According to scaling, ${ }^{54}$ $\Delta E(t) \sim R^{-1}(t)$, and hence $\Delta E(t)$ may be used to indirectly monitor the time-dependent linear length scale of the growth process. In order to obtain reliable ensemble averages, $\Delta E(t)$ is averaged over several independent quenches using different initial configurations and differ- 
ent random-number sequences for the Monte Carlo algorithm. The major part of the nonequilibrium simulations is carried out on lattices of sizes $N=102^{2}$ and $204^{2}$.

\section{A. Quenches to zero temperature}

In the upper panels of Figs. 3 and 4 are shown time series of snapshots of microconfigurations for the $(Q$ $=12)$ - and $(Q=48)$-state model in the case of quenches to $T=0$. Like for the isotropic $Q$-state Potts models ${ }^{19}$ it is found that the morphology of the growing domains becomes more regular and less convoluted as the value of $Q$ is raised. In contrast to the isotropic models, however, the morphology of the boundary network of the models with anisotropic grain-boundary potential has an element of abnormal grain growth ${ }^{43}$ by which conglomerates of small domains are intercalated between larger domains. This feature, which is most pronounced for the $(Q=48)$-state model, cf. Fig. 4 , has led to the concept of wide or soft domain boundaries. ${ }^{39,42}$

A quantitative analysis of the domain-growth process is provided in Figs. 5 and 6 which show that the data for both models are quite accurately described by a power law

$$
\Delta E(t) \sim t^{-n}
$$

over more than two decades. The deviations from the power law at late times are due to finite-size rounding and insufficient statistics. The exponent values derived from Figs. 5 and 6 are $n \simeq 0.36$ for $Q=12$ and $n$ $\simeq 0.24$ for $Q=48$. Our low exponent value for the $(Q$ $=48$ )-state model confirms the results of previous work on this model. ${ }^{42}$ Moreover, the higher exponent value for the $(Q=12)$-state model compares to that found at zero temperature for the Read-Schockley potential ${ }^{42,43}$ with $Q=12$. The Read-Schockley potential is rather similar to that of the present model, Eq. (2), and it also leads to wide domain walls ${ }^{42}$ of the type seen in the upper panels of Figs. 3 and 4 . In the earlier work on the ( $Q=12)$-state model, the growth law and the growth exponent were derived from the domain-size distribution function. Our finding of the same growth exponent value based on the excess energy lends further evidence to the dynamical scaling property of the growth process which implies $^{54}$ that $R(t) \sim \Delta E^{-1}(t)$.

\section{B. Quenches into the cold phase}

Typical series of time evolution of microconfigurations for the $(Q=12)$ - and $(Q=48)$-state models are shown in the lower panels of Figs. 3 and 4 for quenches into the cold phase. It is immediately noted for both models that the morphology of the domain-boundary network is
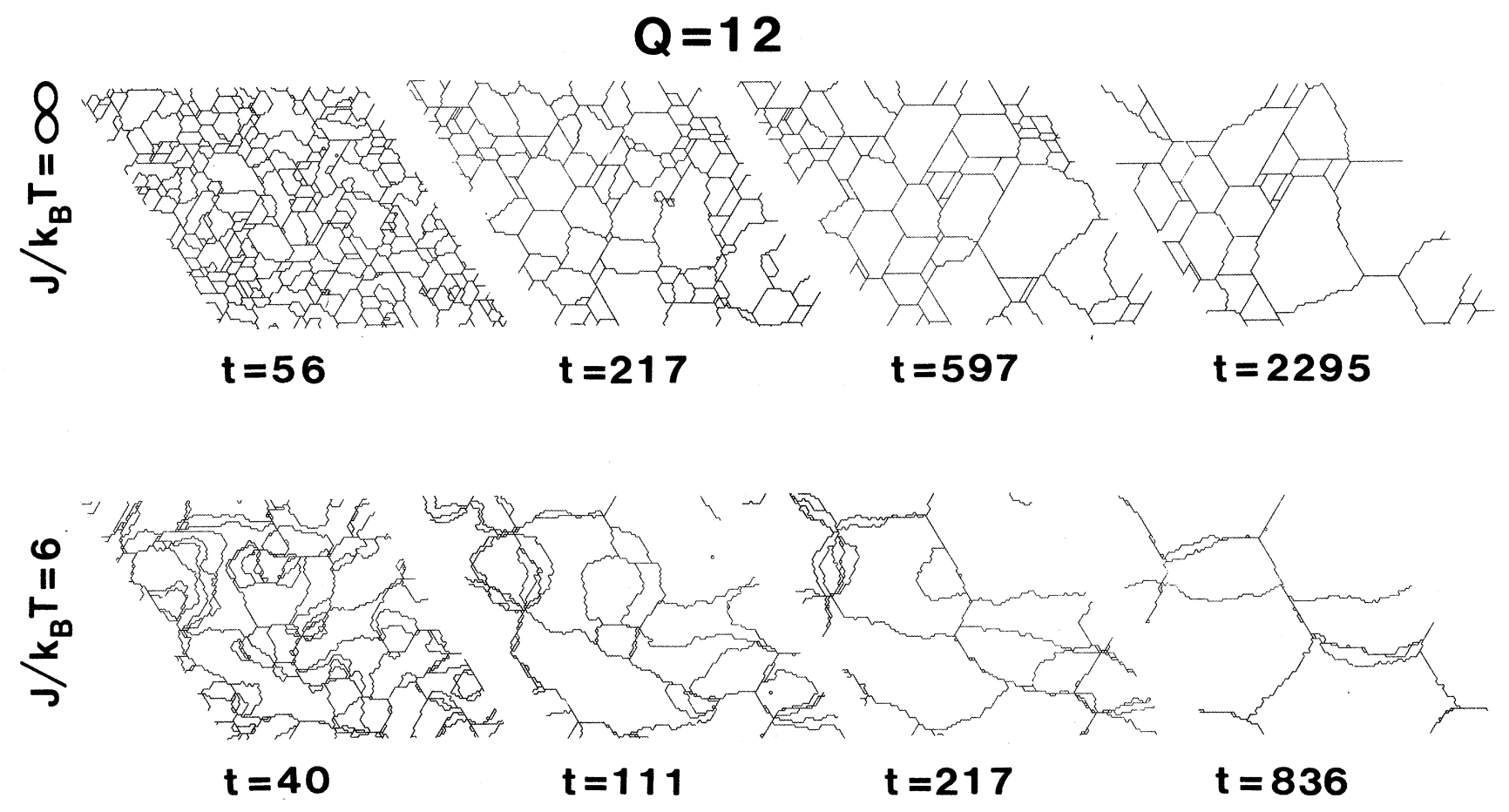

FIG. 3. Snapshots of domain-boundary configurations as they evolve in time for the $(Q=12)$-state anisotropic Potts model on a triangular lattice with $N=102^{2}$ sites. The time parameter $t$ is in units of MCS/S. Results are shown at zero temperature and for a temperature in the cold Potts-ordered phase. 

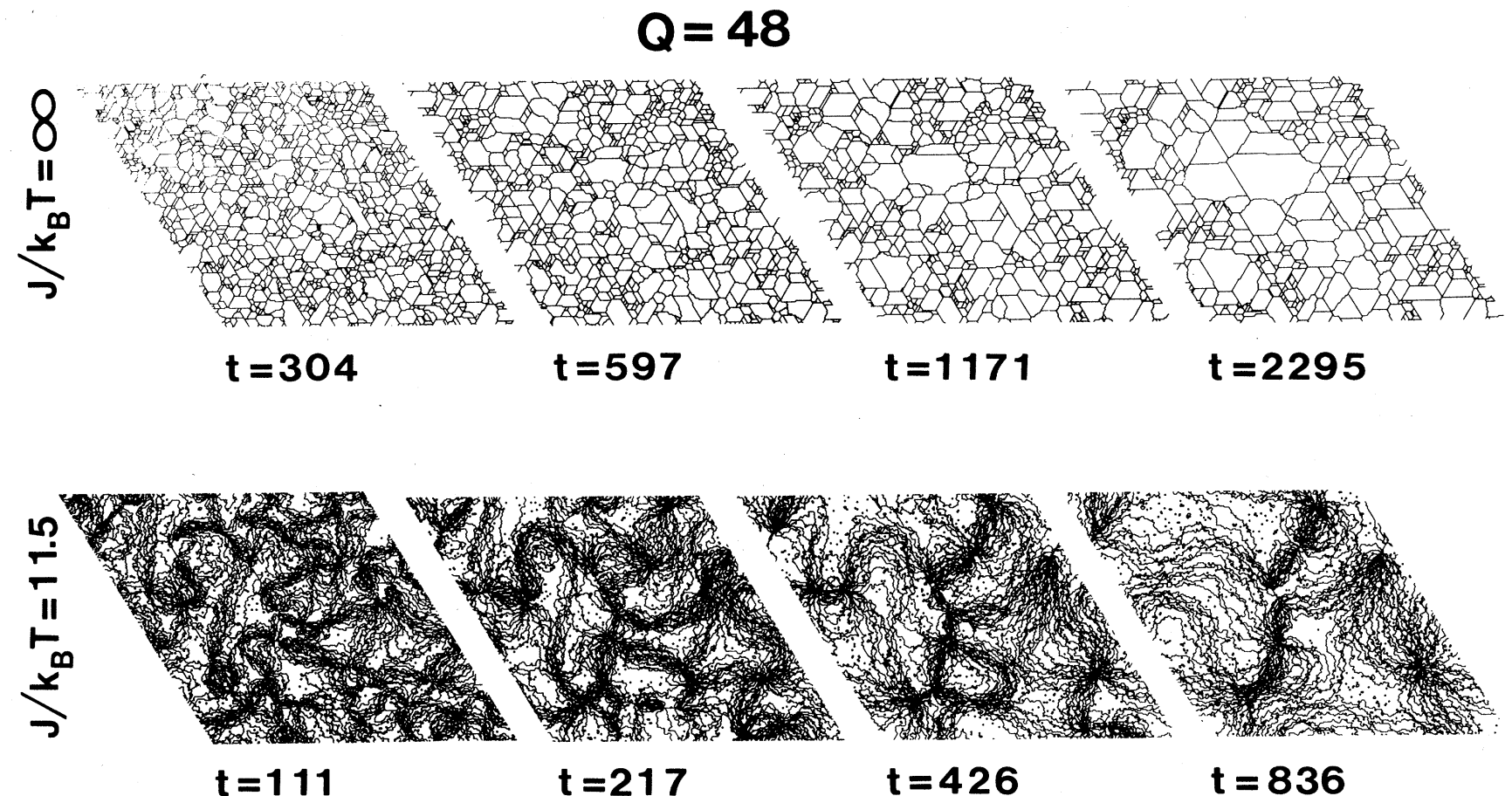

FIG. 4. Snapshots of domain-boundary configurations as they evolve in time for the $(Q=48)$-state anisotropic Potts model on a triangular lattice with $N=204^{2}$ sites. The time parameter $t$ is in units of MCS/S. Results are shown at zero temperature and for a temperature in the cold Potts-ordered phase.

fundamentally different from that at zero growth temperature. Whereas the domains at $T=0$ are compact and regular and associated predominantly with vertices of three rather straight lines, at finite temperatures the domain-boundary network is more irregular. Due to the concave shape of the sine potential the energy per unit length,

$$
\Delta E(q)=|\sin (\pi q / Q)|,
$$

of a domain boundary with $\Delta S=q$ is lower than the energy per unit length,

$$
q \Delta E(1)=q|\sin (\pi / Q)|
$$

of $q$ boundaries with $\Delta S=1$. So the Hamiltonian favors that several domain boundaries coalesce to form a single boundary. The energy gained hereby is very small though, when compared with other energy scales in the Hamiltonian. The smallest gain is

$$
\begin{aligned}
2 \Delta E(1)-\Delta E(2) & =2 \sin (\pi / Q)-\sin (2 \pi / Q) \\
& =(\pi / Q)^{3}+\mathcal{O}\left[(\pi / Q)^{5}\right] .
\end{aligned}
$$

This suggests that the models considered here may have a wetting transition at a temperature $T_{w}$ that scales with $Q$ as

$$
k_{B} T_{w} / J \sim(\pi / Q)^{3}
$$

for large values of $Q$. The finite temperatures quenched to in Figs. 3 and 4 are both in the wetting range of tem-

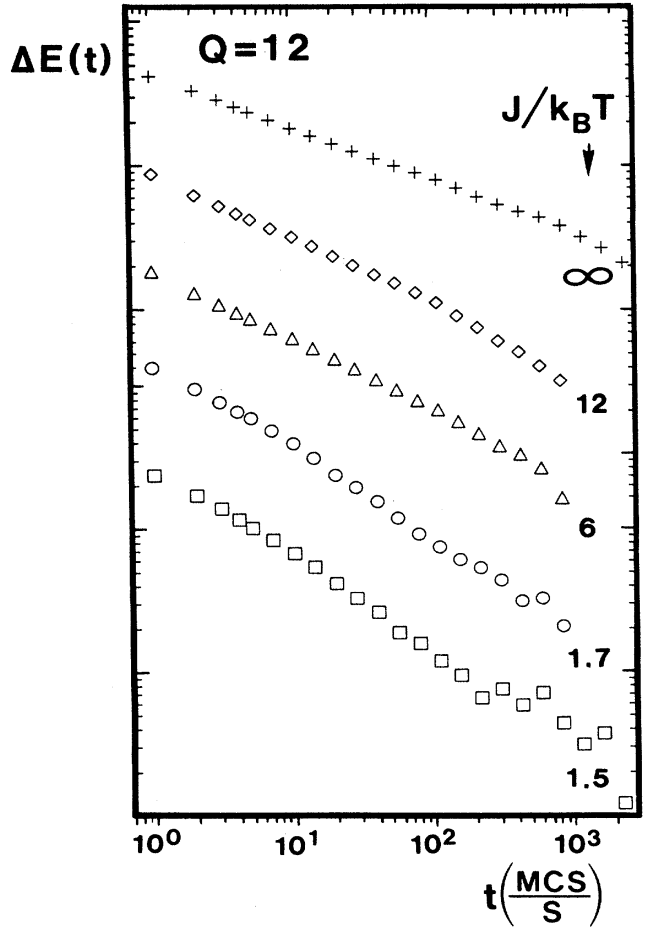

FIG. 5. Double-logarithmic plot of the excess energy, $\Delta E(t)$ in Eq. (6), vs time for the $(Q=12)$-state anisotropic Potts model on a triangular lattice with $N=102^{2}$ sites. The time $t$ is in units of MCS/S. Data are shown for a series of inverse temperatures, $J / k_{B} T$. 


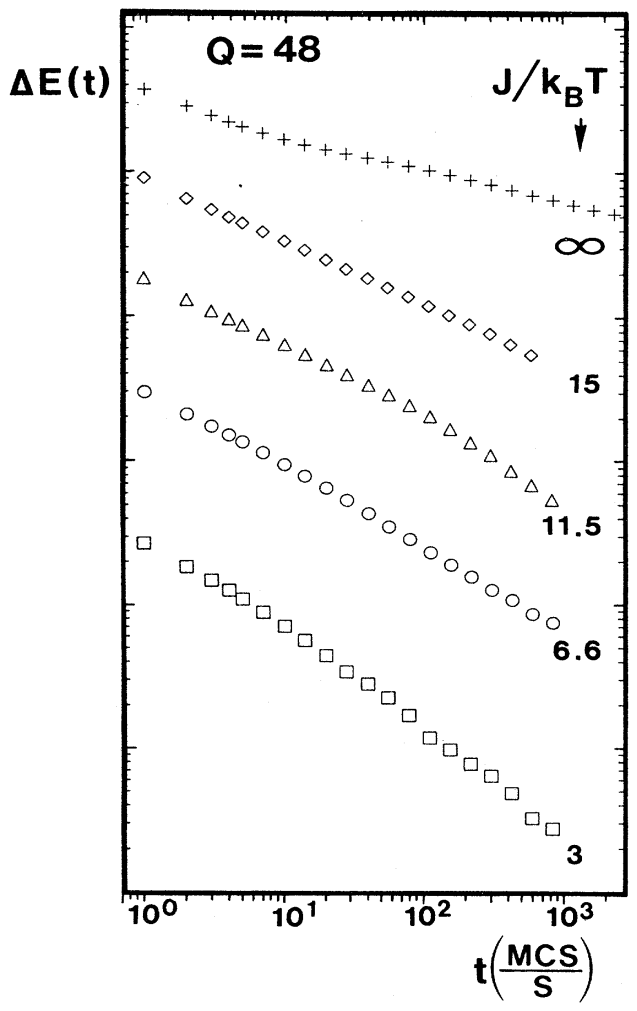

FIG. 6. Double-logarithmic plot of the excess energy, $\Delta E(t)$ in Eq. (6), vs time for the $(Q=48)$-state anisotropic Potts model on a triangular lattice with $N=204^{2}$ sites. The time $t$ is in units of MCS/S. Data are shown for a series of inverse temperatures, $J / k_{B} T$.

peratures. The last snapshot in the lower panel in Fig. 3 shows two long domain boundaries that are wetted, and several small wetted sections of boundaries.

The finite-temperature configurations in Figs. 3 and 4 apparently have a certain degree of vorticity. We have defined a vortex or antivortex center as being a vertex in the domain-boundary network which has the property that the three changes $\Delta S$ in the spin variable encountered in a minimal loop sum to $\pm Q$, once they have been shifted modulo $Q$ to the interval

$$
-Q / 2 \leq \Delta S \leq Q / 2
$$

A minimal loop is defined on the smallest triangles of lattice points around each point of the dual lattice. Because the network is formed by links on a honeycomb lattice, 0 and $\pm Q$ are the only possible values of this sum. With this definition of vortex and antivortex centers we have found that what looks like slowly annihilating vortexantivortex pairs in the lower panels of Figs. 3 and 4 indeed are what they look like. We also found that many other vertices are vortex centers according to our definition, though this is not at all obvious to the eye. This is easy to understand: as opposed to the cosine potential of the standard clock model, the sine potential used here does not make the domain boundaries radiating from a vortex or antivortex center fan out to $Q$ boundaries each having $\Delta S=(-) 1$. Quite to the contrary, any fanning out is entirely due to thermal fluctuations, as argued in the preceding brief discussion of wetting. This is why vortices and antivortices cannot be seen as they can in the standard clock model. It probably also leaves vortices and antivortices with no special status in the ordering dynamics of the models studied here. We rather expect that vortices and antivortices are of special relevance for an understanding of the ordering dynamics only in models with potentials that make these configurations local minima of the Hamiltonian.

Comparing the lower panels in Figs. 3 and 4 we observe that the presence of vortices and antivortices in the ordering process is much more pronounced for the larger value of $Q$. This was found also for the standard $Q$-state clock models. ${ }^{46,47}$ By comparison with typical equilibrium configurations in the cold phase we conclude that the vortices seen in Figs. 3 and 4 are distinct features of the nonequilibrium ordering process and are not typical thermal equilibrium excitations in the Potts-ordered phase.

We have applied our definition of vortex and antivortex centers to the zero-temperature configurations shown in the upper panels of Figs. 3 and 4 , and found that a finite fraction of the vertices of the boundary network are vortices and antivortices. This is not surprising: even if the values of the three spin variables surrounding a vertex were chosen at random, a finite fraction of vertices would be vortices and antivortices.

Neither for quenches to finite temperatures nor to zero temperature did we observe pinning of the ordering process by vortices and antivortices. Instead we observed them disappearing by mutual annihilation. This is seen to happen in Figs. 3 and 4 to the visible vortex-antivortex pair. This is similar to what has been found in finitetemperature quenches of the clock models. ${ }^{47}$ However, vortex pinning has been observed in these models ${ }^{46}$ to slow down the growth at long times in the case of zerotemperature quenching.

In Figs. 5 and 6 are shown the $\Delta E(t)$ data for quenches

TABLE I. Kinetic growth exponent $n$ as a function of growth temperature for the $(Q=12)$ state Potts model with anisotropic grain-boundary potential.

\begin{tabular}{lccccc}
\hline \hline \multirow{2}{*}{ Zero temperature } & \multicolumn{2}{c}{ Cold phase } & \multicolumn{2}{c}{ Intermediate phase } \\
$J / k_{B} T$ & $\infty$ & 12 & 6 & 1.7 & 1.5 \\
\hline$n$ & 0.36 & 0.46 & 0.50 & 0.65 & 0.61 \\
\hline \hline
\end{tabular}


TABLE II. Kinetic growth exponent $n$ as a function of growth temperature for the $(Q=48)$ state Potts model with anisotropic grain-boundary potential.

\begin{tabular}{lccccc}
\hline \hline & Zero temperature & \multicolumn{2}{c}{ Cold phase } & \multicolumn{3}{c}{ Intermediate phase } \\
$J / k_{B} T$ & $\infty$ & 15 & 11.5 & 6.6 & 3 \\
\hline$n$ & 0.24 & 0.44 & 0.51 & 0.55 & 0.69 \\
\hline \hline
\end{tabular}

into the cold phase. From these data we make the striking observation that the growth is still described by a power law but now with an exponent that is close to the classical Lifshitz-Allen-Cahn value, $n=\frac{1}{2}$. The numerical values of $n$ are compiled in Tables I and II for the two models. Approximately the same value of $n$ is found to hold for both the $(Q=12)$ - and the $(Q=48)$-state models, and the exponent value is moreover independent of temperature within the cold phase. The data of Figs. 5 and 6 support the conclusion that vortex formation does not lead to a pinning of the growth in the cold phase.

\section{Quenches into the intermediate phase}

Since we have not attempted to characterize the intermediate phase by an order parameter, it is not possible to illustrate unambiguously the ordering process by snapshots. However, it is instructive to study configurations as they result from quenches into the intermediate phase by applying the same domain-boundary criterion as for the cold Potts-ordered phase. In Fig. 7 is shown a configuration for the $(Q=12)$-state model at some late time after a quench into the intermediate phase. The pattern of domains of different Potts order is extremely convoluted and rather large domains have evolved. A similar convoluted pattern is found for quenches of the $(Q=48)$ state model into the intermediate phase, but at a given time the domains in this model are much smaller than those in the $(Q=12)$-state model.

The growing domain patterns for quenches into the

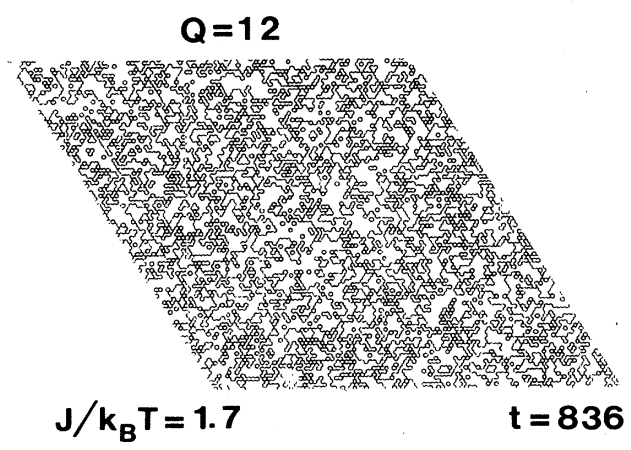

FIG. 7. Snapshot of a domain-boundary configuration for the $(Q=12)$-state anisotropic Potts model on a triangular lattice at time $t=836 \mathrm{MCS} / \mathrm{S}$ after a quench from $T=\infty$ to a temperature $J / k_{B} T=1.7$ within the intermediate KosterlitzThouless phase. Domain boundaries between regions of different Potts order are shown. The results are obtained from Monte Carlo simulations on a lattice with $N=102^{2}$ sites. intermediate phase have no discernible vortex configurations of the type seen in quenches to the cold phase. Using our definition of vortex and antivortex centers we found that they are there, however, in tightly bound pairs, just like they are in equilibrium configurations in the intermediate phase of the standard clock model and in the Kosterlitz-Thouless phase of the $X Y$ model.

In Figs. 5 and 6 are shown the $\Delta E(t)$ data for quenches into the intermediate phase. It is not clear that $\Delta E(t)$ in this phase is a good measure of the time dependence of a linear length scale since the scaling argument of Binder and Stauffer ${ }^{54}$ does not necessarily carry over to phases with topological ordering. Nevertheless, the data in Figs. 5 and 6 suggest that the $\Delta E(t)$ data effectively obey a power law in time and that the exponent value $n$ is somewhat above $\frac{1}{2}$; cf. Tables I and II. When judging the implications of this apparent deviation from the classical Lifshitz-Allen-Cahn growth, it should be kept in mind that the locations of the phase boundaries of the two models are subject to some uncertainty. In particular, it is not unlikely that both quench temperatures in the intermediate phase for the $(Q=12)$-state model as well as the highest quench temperature in the intermediate phase for the $(Q=48)$-state model are close to the phase transition temperatures. In that case our statistics may be too poor to describe the true dynamic behavior.

\section{DISCUSSION}

We have presented a study of the dynamics of the ordering processes in two-dimensional $Q$-state Potts models with an anisotropic grain-boundary potential, Eq. (2), using Monte Carlo computer-simulation techniques. The ground-state ordering is highly degenerate. The two particular models studied, $Q=12$ and 48 , have a phase structure which involves two thermal phase transitions which separate an intermediate phase from a lowtemperature Potts-ordered phase and a high-temperature disordered phase.

The main results of the simulations show that the domain-growth dynamics at zero temperature are described by an algebraic growth law with an anomalously low value of the growth exponent, $n$. For $Q=48$, the exponent value is $n \simeq 0.24$ in accordance with earlier work. ${ }^{42,43}$ The $(Q=48)$-state model is characterized by a tendency to evolve wide domain walls; cf. Fig. 4. The wide domain walls contain a conglomerate of small domains of different Potts order. It has been suggested $15,39,42$ that the zero-temperature growth of models which support soft or wide domain walls may 
belong to a special dynamical universality class characterized by $n \simeq \frac{1}{4}$, independent of ordering degeneracy, $p$, and the degree of wall softness. This suggestion has been questioned by other authors. ${ }^{12,40}$ The other softwall models which give rise to $n \simeq 0.25$ at zero temperature include the herringbone model ${ }^{39}(p=6)$, the $X Y$ model with cubic anisotropy ${ }^{15,20,39}(p=4)$, and the $X Y$ model with uniaxial anisotropy ${ }^{15,20,39,55}(p=2)$. The soft domain walls in these models correspond to a gradient in the order parameter going from one domain to the other. It was recently shown ${ }^{15,36}$ that the growth behavior described by $n \simeq 0.25$ in these models is restricted to very low temperatures, possibly to $T=0$ exclusively, and that there is a distinct crossover to the Lifshitz-AllenCahn exponent value $n=\frac{1}{2}$ at finite growth temperatures. The finding in this work of a similar crossover corroborates the general validity of this result. In a recent theoretical analysis of the detailed microscopic motion of the different domain-wall types, Castán and Lindgaard ${ }^{55}$ have provided theoretical support for the special zerotemperature dynamical universality class.

The dynamical ordering processes in the high- $Q$ Potts models with anisotropic grain-boundary potential demonstrate distinct vortex formation involving vortexantivortex pairs of many rays at low, finite temperatures; cf. Figs. 3 and 4 . This vortex formation does not lead to a pinning of the growth process. The same lack of pinning was found at finite temperatures from Monte Carlo quenching experiments on the high- $Q$ clock models. ${ }^{47}$ Similarly, computer simulation of the domain-growth dynamics in Ising models with competing interactions and nonconserved order parameter, which leads to vortex formation in $(4 \times 1)$ phases $(p=4)$, has demonstrated that vortex pinning does not become operative and the Lifshitz-Allen-Cahn growth-exponent value, $n=\frac{1}{2}$, applies. These findings are supported by a theory for $Q=p \geq 3$ clock models due to Kawasaki ${ }^{56}$ (and extensions thereof ${ }^{16}$ ) who considers the coupling between vertices and domain boundaries. Kawasaki reduces the problem of the interface dynamics to that of dissipative dynamics of opposite Coulomb charges. It is then found for any value of $p$, at sufficiently late times, that the Lifshitz-Allen-Cahn exponent value should apply and vortex pinning does not influence the growth law. It is not obvious, however, how this theory may be generalized to be valid for anisotropic Potts models. ${ }^{56}$

The ordering dynamics in the $Q$-state Potts models with anisotropic grain-boundary potential is complicated by the fact that these models, for large $Q$, have a Kosterlitz-Thouless-type phase at intermediate temperatures. In the present paper we have shown for the potential in Eq. (2) that even in this phase is the ordering dynamics effectively described by algebraic growth laws with exponent values which are somewhat larger than $\frac{1}{2}$. Considering the possible influence of critical fluctuations at these quench temperatures we are unable to decide whether or not our exponent values are actually inconsistent with classical behavior. We are not aware of other computer simulations of ordering dynamics in KosterlitzThouless phases.

The high- $Q$ Potts models with anisotropic grainboundary potential have been proposed as models of grain growth in polycrystalline materials. ${ }^{43}$ However, our finding of a grain distribution with a morphology involving vortex structures may well disqualify this model as a model of real materials at finite temperatures. In fact our results lead us to suggest that realistic anisotropic grainboundary potentials should have a pronounced superlinear character, possible logarithmic, ${ }^{43}$ in order to suppress the fluctuation-induced wetting phenomena. Furthermore, the result by Grest et al. ${ }^{43}$ of the anisotropy as leading to anomalously low growth exponent values is, from the present work, concluded to apply only at very low temperatures, possibly only at zero temperature.

\section{ACKNOWLEDGMENTS}

It is a pleasure to thank Dieter W. Heermann and David J. Srolovitz for stimulating discussions on the nonequilibrium dynamics of systems undergoing ordering processes. This work was supported by the Danish Technical Research Council under Grant No. J.nr.16-4425.K and the Danish Natural Science Research Council under Grant No. J.nr.5.21.99.72.
${ }^{1}$ J. D. Gunton, M. San Miguel, and P. S. Sahni, in Phase Transitions and Critical Phenomena, edited by C. Domb and J. L. Lebowitz (Academic, New York, 1983), Vol. 8, p. 267.

${ }^{2} \mathrm{~J}$. D. Gunton, in Time-Dependent Effects in Disordered Materials, edited by R. Pynn and T. Riste (Plenum, New York, 1987), p. 387.

${ }^{3}$ J. G. Amar, F. E. Sullivan, and R. D. Mountain, Phys. Rev. B 37, 196 (1988).

${ }^{4}$ T. M. Rogers, K. R. Elder, and R. C. Desai, Phys. Rev. B 37, 9638 (1988).

${ }^{5}$ A. Sadiq and K. Binder, J. Stat. Phys. 35, 517 (1984).

${ }^{6}$ A. Chakrabarti, J. B. Collins, and J. D. Gunton, Phys. Rev. B 38, 6894 (1988).

${ }^{7}$ Y. Oono and S. Puri, Phys. Rev. A 38, 434 (1988); S. Puri and Y. Oono, ibid. 38, 1542 (1988).

${ }^{8}$ Z. W. Lai, G. F. Mazenko, and O. T. Valls, Phys. Rev. B 37, 9481 (1988).

${ }^{9}$ P. C. Hohenberg and B. I. Halperin, Rev. Mod. Phys. 49, 435 (1977).

${ }^{10}$ K. Kaski, T. Ala-Nissilä, and J. D. Gunton, Phys. Rev. B 31, 310 (1985); T. Ala-Nissilä, J. D. Gunton, and K. Kaski, ibid. 33, 7583 (1986).

${ }^{11} \mathrm{~J}$. Viñals and J. D. Gunton, Surf. Sci. 157, 473 (1985).

${ }^{12}$ A. Milchev, K. Binder, and D. W. Heermann, Z. Phys. B 63, 521 (1986).

${ }^{13}$ S. Kumar, J. D. Gunton, and K. Kaski, Phys. Rev. B 35, 8517 (1987).

${ }^{14}$ A. Høst Madsen, P. J. Shah, T. V. Hansen, and O. G. Mouritsen, Phys. Rev. B 36, 2333 (1987). 
${ }^{15}$ O. G. Mouritsen and E. Praestgaard, Phys. Rev. B 38, 2703 (1988).

${ }^{16}$ T. Ala-Nissilä, J. D. Gunton, and K. Kaski, Phys. Rev. B 37, 179 (1988).

${ }^{17}$ G. N. Hassold and D. J. Srolovitz, Phys. Rev. B 37, 3467 (1988).

${ }^{18}$ H. C. Fogedby and O. G. Mouritsen, Phys. Rev. B 37, 5962 (1988).

${ }^{19}$ G. S. Grest, M. P. Anderson, and D. J. Srolovitz, Phys. Rev. B 38, 4752 (1988).

${ }^{20}$ P.-A. Lindgaard, H. E. Viertiö, and O. G. Mouritsen, Phys. Rev. B 38, 6798 (1988).

${ }^{21}$ M. K. Pahni, J. L. Lebowitz, M. H. Kalos, and O. Penrose, Phys. Rev. Lett. 45, 366 (1980).

${ }^{22}$ A. Milchev, D. W. Heermann, and K. Binder, Acta Metall. 36, 377 (1988).

${ }^{23}$ R. Toral, A. Chakrabarti, and J. D. Gunton, Phys. Rev. Lett. 60, 2311 (1988).

${ }^{24}$ C. Roland and M. Grant, Phys. Rev. Lett. 60, 2657 (1988).

${ }^{25}$ E. Gawlinski, J. D. Gunton, and J. Viñals (unpublished).

${ }^{26}$ C. Roland and M. Grant, Phys. Rev. B 39, 11971 (1989).

${ }^{27}$ G. S. Grest and P. S. Sahni, Phys. Rev. B 30, 226 (1984).

${ }^{28}$ G.-C. Wang and T.-M. Lu, Phys. Rev. Lett. 50, 2014 (1983).

${ }^{29}$ M. C. Tringides, P. K. Wu, and M. G. Lagally, Phys. Rev. Lett. 59, 315 (1987).

${ }^{30} \mathrm{~S}$. Komura, K. Osamura, H. Fujii, and T. Takeda, Phys. Rev. B 31, 1278 (1985).

${ }^{31}$ S. Katano, M. Iizumi, R. M. Nicklow, and H. R. Child, Phys. Rev. B 38, 2659 (1988).

${ }^{32}$ S. E. Nagler, R. F. Shannon, C. R. Harkless, M. A. Singh, and R. M. Nicklow, Phys. Rev. Lett. 61, 718 (1988).

${ }^{33}$ I. M. Lifshitz, Zh. Eksp. Teor. Fiz. 42, 1354 (1962) [Sov. Phys.-JETP 15, 939 (1962)].

${ }^{34}$ S. M. Allen and J. W. Cahn, Acta Metall. 27, 1085 (1979).

${ }^{35}$ I. M. Lifshitz and V. V. Sloyzov, J. Phys. Chem. Solids 19, 35 (1961).

${ }^{36}$ O. G. Mouritsen, H. C. Fogedby, and E. Praestgaard, in $D y$ - namics of Ordering Processes in Condensed Matter, edited by S. Komura (Plenum, New York, 1988), p. 133.

${ }^{37} \mathrm{~J}$. Viñals and M. Grant, Phys. Rev. B 36, 7036 (1987).

${ }^{38} \mathrm{~J}$. Viñals and J. D. Gunton, Phys. Rev. B 33, 7795 (1986).

${ }^{39}$ O. G. Mouritsen, Phys. Rev. Lett. 56, 850 (1986); Phys. Rev. B 31, 2613 (1985); 28, 3150 (1983).

${ }^{40}$ W. van Saarloos and M. Grant, Phys. Rev. B 37, 2274 (1988); O. G. Mouritsen and E. Praestgaard, ibid. 37, 2278 (1988).

${ }^{41}$ J.-K. Zuo, G.-C. Wang, and T.-M. Lu, Phys. Rev. Lett. 60, 1053 (1988).

${ }^{42}$ G. S. Grest, D. J. Srolovitz, and M. P. Anderson, Phys. Rev. Lett. 52, 1321 (1984).

${ }^{43}$ G. S. Grest, D. J. Srolovitz, and M. P. Anderson, Acta Metall. 33, 509 (1985).

${ }^{44}$ J. L. Cardy, J. Phys. A 13, 1507 (1979).

${ }^{45} \mathrm{~K}$. Kaski and J. D. Gunton, Phys. Rev. B 28, 5371 (1983).

${ }^{46}$ G. S. Grest and D. J. Srolovitz, Phys. Rev. B 30, 6535 (1984).

${ }^{47}$ K. Kaski, M. Grant, and J. D. Gunton, Phys. Rev. B 31, 3040 (1985).

${ }^{48}$ O. G. Mouritsen, Computer Studies of Phase Transitions and Critical Phenomena (Springer-Verlag, New York, 1984).

${ }^{49}$ D. W. Heermann, Computer Simulation Methods (SpringerVerlag, New York, 1986).

${ }^{50}$ M. Creutz, L. Jacobs, and C. Rebbi, Phys. Rev. Lett. 42, 1390 (1979); Phys. Rev. D 20, 1915 (1979).

${ }^{51}$ J. Tobochnik, Phys. Rev. B 26, 6201 (1982).

${ }^{52}$ M. S. S. Challa and D. P. Landau, Phys. Rev. B 33, 437 (1986).

${ }^{53}$ J. Tobochnik and G. V. Chester, Phys. Rev. B 20, 3761 (1979).

${ }^{54}$ K. Binder and D. Stauffer, Phys. Rev. Lett. 33, 1006 (1974).

${ }^{55}$ T. Castán and P.-A. Lindgaard, Phys. Rev. B 40, 5069 (1989).

${ }^{56}$ K. Kawasaki, Phys. Rev A 31, 3880 (1985). 